\title{
Relaciones entre Estados Unidos y Ecuador en el ámbito de la seguridad durante la Segunda Guerra Mundial
}

\section{The Relations between the United States and Ecuador in the field of Security during World War II}

\author{
César Castilla \\ Université Toulouse 1 Capitole \\ Grupo de trabajo CLACSO en Medio Oriente y Norte de África \\ Quito, Ecuador \\ (D) https://orcid.org/0000-0002-5368-3912 \\ ccpublications99@gmail.com \\ Karol Tituaña \\ Universidad de Los Hemisferios \\ Quito, Ecuador \\ (D) https://orcid.org/0000-0002-3254-2758 \\ karolfer24797@gmail.com
}

\section{Resumen}

Las primeras actividades conjuntas en el campo de la seguridad entre Ecuador y Estados Unidos se dan en territorio ecuatoriano durante la Segunda Guerra Mundial, y corresponden a un nuevo enfoque americano de seguridad donde se privilegia la conformación de estructuras mixtas en el campo de las relaciones internacionales. Esto significó acciones de control y restricción de la entrada de ciudadanos provenientes de los países del eje al país sudamericano, el intercambio de información de extranjeros sospechosos en las embajadas, la aplicación de la inteligencia de señales, la presencia de agentes del FBI y la confección de una lista negra, entre otras. Con esta nueva visión de seguridad, la hegemonía norteamericana no 
solamente prevalecería en Latinoamérica, sino también en la parte occidental de Europa al finalizar la Segunda Guerra Mundial. El presente artículo ha recopilado fuentes primarias del archivo histórico de la Cancillería del Ecuador; y tiene como objetivo general identificar las causas del trabajo conjunto entre Ecuador y Estados Unidos en el campo de la seguridad durante la Segunda Guerra Mundial a través de una aproximación teórica.

Palabras clave: Ecuador; Estados Unidos; nazismo; seguridad internacional; relaciones internacionales.

\begin{abstract}
The first joint activities between Ecuador and the United States in the field of international security took place in ecuadorian territory during WWII. This is a novel approach adopted by the americans that has privileged the formation of mixed structures in the realm of international relations. That meant actions such as the control and restriction of the entry of axis nationals to Ecuador, the exchange of information on suspected foreigners working at embassies, the application of intelligence plans like the signal intelligence, $\mathrm{FBI}$ agents in Ecuador and the application of a blacklist, among others. Thanks to this new international security approach, the hegemony of the united states would prevail in Latin America and western Europe at the end of WWII. This academic paper has collected first-hand sources from the historical archive of the ecuadorian chancellery. This research aims to identify the causes of the joint activities between Ecuador and the United States in international security issues during wwii through an ir's theoretical approach.
\end{abstract}

Keywords: Ecuador; United States; nazism; international security; international relations.

Recibido: 11/06/2020 Aceptado: 20/08/2021

\title{
Introducción
}

Al iniciar el siglo XX el sistema internacional es eurocéntrico y la potencia indiscutible es Inglaterra. No obstante, en la región del Asia Pacífico, Japón es la nación con mayor grado de desarrollo. Ante esta situación, los norteamericanos no pueden quedarse fuera de la competencia e intentan hacerse un lugar dentro de la arena internacional. Al ser Estados Unidos un país liberal tiene limitaciones para ejercer un poder coercitivo en la región de manera directa, pero la tendencia de liderazgo estaba marcada desde la doctrina Monroe enunciada en 1823, cuando los americanos proyectan el control sobre América Latina. Posteriormente, esto se evidencia durante el 
mandato de Theodore Roosevelt, con su doctrina del Big Stick desde 1901, a partir de entonces los países centroamericanos son los que sufrieron las consecuencias de la diplomacia americana. Esto ocasiona un desgaste en la imagen de los americanos.

Por entonces, América Latina tiene una marcada influencia americana en lo político y económico. No obstante, en el periodo de entre guerras (1919-1939) los europeos se habían interesado también por la región, en especial la Alemania Nazi que logró despertar la simpatía de diferentes gobiernos de América del Sur, como Argentina, Chile, Perú entre otros. Al inicio del conflicto en septiembre de 1939, los gobiernos latinoamericanos optan por una posición neutral en torno al conflicto. Conforme transcurrieron los primeros años de combate y la entrada de los Estados Unidos a la guerra en diciembre de 1941, emergen algunos aliados importantes como Brasil, que debido a su posición geoestratégica envía tropas hacia Europa. De la misma manera, Estados Unidos encuentra otros aliados importantes en Centroamérica, como Guatemala, y en América del Sur, al Ecuador, durante los últimos años de esta conflagración mundial.

Las relaciones entre Ecuador y Estados Unidos durante la Segunda Guerra Mundial resaltan la importancia del campo de la seguridad en el ámbito de las relaciones internacionales. El siglo XX implica una evolución sobre el concepto de la seguridad, donde los Estados Unidos han sido los pioneros dentro de este campo. Un claro ejemplo son las primeras actividades conjuntas con Ecuador, durante la segunda mitad de la década de 1930, para alejar la influencia de la Alemania Nazi en la región. Para ello, los americanos estimulan a un país con una serie de ofrecimientos, entre ellos, préstamos, capacitaciones, etc.; con la finalidad de obtener respaldo en este ámbito.

Es así como en este periodo, Estados Unidos aplica un nuevo enfoque de seguridad que parece darle muy buenos resultados no solamente durante la Segunda Guerra Mundial sino también en un escenario de post guerra; es decir desde 1946 hasta 1991 durante la Guerra Fría. En esta etapa, se puede apreciar que los decisores americanos buscan un punto de equilibrio sustentado en el crecimiento económico, pero también en el de la seguridad. Dicho enfoque fue expandido a lo largo del continente americano. 
Por el lado del Ecuador, este país cuenta con una economía exportadora de materias primas. Políticamente, en la primera mitad de la década de 1930 tuvo gobiernos que habían mostrado simpatías con el Tercer Reich.

Posteriormente, los americanos, al ver sus bases militares de Pearl Harbor atacadas por los japoneses el 7 de diciembre de 1941; y con el objetivo de minimizar sus pérdidas en este escenario de guerra, optan por tercerizar el riesgo mediante la conformación de una coalición de tropas latinoamericanas que combatiese juntos a los aliados. Es así como se acercan a Ecuador y ejercen influencia en los planos económico y político.

La metodología de la presente investigación es de carácter cualitativo dado que se pretende reconstruir la agenda americana en Ecuador sobre temas de seguridad durante la Segunda Guerra Mundial, basada en fuentes primarias del Archivo Histórico de la Cancillería del Ecuador y, secundarias, como libros o artículos especializados. A pesar de las distintas visiones que existen sobre las relaciones internacionales, como la Norte-Sur, en este trabajo se adopta un análisis estructural apoyado en la teoría neorrealista de las relaciones internacionales de Kenneth Waltz (1979) para explicar las pretensiones hegemónicas americanas en Ecuador y la región. Además, de complementarlo con la "teoría del juego" planteada por John Von Neumann and Oskar Morgenstern (1953) donde se analizan las reglas, las movidas y la elección para determinar si la estrategia implícita en esta relación durante la Segunda Guerra Mundial fue de suma cero, ganar-ganar o perder-perder.

\section{Aproximaciones a la seguridad en el marco de la Segunda Guerra Mundial}

Entender la seguridad de una nación siempre ha sido el gran dilema de emperadores, monarcas, presidentes, dictadores, entre otros. Seguridad es un término que deriva del latín Securitas, y según el diccionario de la RAE, significa Libre y exento de todo peligro, daño y riesgo ${ }^{1}$.

No obstante, a partir de la Edad Moderna, este término comienza a evolucionar y cobra una gran importancia en el mundo contemporáneo. La Paz de Westfalia, lograda a través de los tratados de Osnabrück y Münster en octubre de 1648, pone fin a la guerra de los Treinta Años y la guerra de

${ }^{1}$ Diccionario de la Real Academia Española (RAE). https://dej.rae.es/lema/seguridad 
los Ochenta Años. Es el inicio de una nueva era en el campo de las relaciones internacionales, no solamente porque se está ante el debut del rol que jugaría el Estado Nación en Europa, sino que incorpora una visión de seguridad estado-céntrica por la marcada influencia de Niccolò di Bernando dei Machiavelli (1469-1527) en los monarcas de aquel entonces. Es necesario indicar que para Maquiavelo la seguridad era un objetivo alcanzable mediante la unión de todos; desde su perspectiva que los hombres no son ni buenos ni malos.

La paz de Westfalia implica que la seguridad se centre en la defensa de las fronteras de las monarquías, obviamente bajo un interés común. Esto se convertiría en el común denominador durante las siguientes décadas, hasta que Napoleón Bonaparte irrumpe a inicios del siglo XIX y desestabiliza al antiguo régimen europeo. No obstante, este nuevo orden no duraría mucho $y$, poco después de su derrota en la batalla de Waterloo en junio 1815, el Congreso de Viena (septiembre 1814-junio 1815) restablece el antiguo régimen pro-absolutista.

Esto constituye otro cambio en el paradigma de las relaciones internacionales, no solo por su interés de frenar al ejército francés sino porque este constituye un legado bastante interesante en el campo de la seguridad dado que de aquí se deriva la Santa Alianza (septiembre 1815), es decir una unión militar con valores cristianos conformada por Austria, Prusia y Rusia. Esto significa que las tres monarquías conciben una visión colectiva de la seguridad para enfrentar al enemigo francés. Si bien es cierto que cuando se hablaba de seguridad la visión predominante era la de Maquiavelo, no obstante, el general prusiano Carl von Clausewitz (1780-1831) complementa la visión del primero y desarrolla el concepto de defensa. Para tales efectos Clausewitz argumenta que la defensa consiste en repeler un golpe cuya característica es la espera de ese golpe. Lo que considera como un acto defensivo, y solo por este rasgo la defensa puede separarse del ataque en la guerra. Pero dado que una defensa absoluta contradice completamente el concepto de guerra, porque solo uno emprende la guerra, entonces la defensa también se puede usar en la guerra (Clausewitz, 1832, p. 247).

Esta nueva tendencia de seguridad colectiva enfocada en la defensa predomina en las naciones europeas especialmente de 1890 a 1914. Por un lado, la Triple Alianza trata de aislar diplomáticamente a Francia y obstaculizar su expansión colonial; mientras que la Triple Entente, alianza militar de Francia, Reino Unido y de Rusia Imperial se configura con la 
finalidad de persuadir a los alemanes de desistir de cualquier intento de agresión en contra de estos países. En 1871, Prusia había derrotado a los franceses en el marco de las guerras franco-prusianas por lo que se preveía una expansión del Segundo Reich. Cabe resaltar que la Triple Entente se crea tras la firma de una convención militar entre Francia y Rusia el 27 de diciembre de 1893 y posteriormente con la firma entre Francia y el Reino Unido de la Entente Cordiale, el 8 de abril de 1904.

A la siguiente década la humanidad presenciaría el inicio de la Primera Guerra Mundial. Según Hobsbawm (1999, p. 33), esta comenzó como una guerra esencialmente europea entre la Triple Alianza y las potencias centrales, que se extendió hacia Extremo Oriente y el Pacifico Occidental, donde Estados Unidos tuvo un rol decisivo. Sin olvidar que la ideología liberal y la nacionalista se enfrentarían creando estructuras de seguridad en el sistema internacional. Posteriormente, el mismo enfoque de seguridad colectiva se emplearía en el marco de la Segunda Guerra Mundial.

En los años '30 del siglo $\mathrm{XX}$, la seguridad sufre otra transformación alejándose de su visión colectiva. ¿Qué cambia? Conforme se industrializan las economías, algunos Estados como el americano, se percatan que no es suficiente reforzar sus fronteras, ni contar con un apoyo solidario de otro Estado en caso de una amenaza exterior, sino que otros factores, como el económico, también contribuyen a la seguridad de un Estado.

\section{Enfoque de seguridad americano}

En virtud de lo expuesto, en el siguiente apartado se identifican y analizan las estructuras económicas internas y externas que intervienen en la perspectiva de seguridad desplegada por Estados Unidos.

\section{Estructuras económicas internas}

Algunas potencias involucradas en la Segunda Guerra Mundial integran muy bien lo económico al campo de la seguridad, como los Estados Unidos y la Alemania nazi.

Un suministro constante de mano de obra es importante para cualquier economía de guerra; muy pocos trabajadores o muy pocos soldados predicen una eventual derrota en el frente de batalla. Durante la Segunda Guerra Mundial, la mano de obra importada ayudó a sostener 
las economías de Alemania y Gran Bretaña. Alemania utilizó el trabajo forzado y voluntario extranjero de la Europa ocupada para reemplazar a los trabajadores alemanes enviados a los frentes (Golson, 2019).

Con respecto a Estados Unidos, aprovecha la mano de obra migrante para sustentar su economía; es decir de aquellos que escapan principalmente de Europa.

\section{Estructuras económicas externas}

Con el objetivo de reforzar su seguridad y asegurar su supervivencia, Estados Unidos se vale de la creación de estructuras económicas externas. El negocio americano de vender armas a los países involucrados en la Segunda Guerra Mundial no solamente crea una dependencia de estos actores hacia los americanos, sino también permite que estos se consoliden como una potencia armamentista. El presidente Franklin D. Roosevelt (1933-1945), desde que asume el poder, atraviesa un sistema internacional completamente inestable, dado que la Sociedad de Naciones se debilita progresivamente al no contar con un poder coercitivo. Por tanto, no puede controlar a algunos actores como Japón, Italia y desde luego a Alemania Nazi que rompen el orden idealista de esta organización. Dentro de este contexto, Roosevelt critica públicamente el accionar de Japón en octubre de 1937, y anuncia un plan para que continúe el aprovisionamiento de armas desde los Estados Unidos hacia China a pesar de la existencia del Acta de Neutralidad (Stuart, 2008, p.29).

Además, Estados Unidos aprovecha el incipiente proceso de integración americano, iniciado con la I Conferencia Interamericana (1890), cuando se crea la Unión Internacional de las Repúblicas Americanas con una Oficina Comercial y aprovecha su dominio sobre el sistema de cooperación interamericano con su doctrina de "seguridad económica". Con el transcurrir del tiempo, Estados Unidos haría lo mismo con otras instituciones, como el Banco Interamericano y el Comité Consultivo Económico y Financiero Interamericano, que formarían parte de su estrategia para influenciar algunos Estados de la región como es el caso de Ecuador.

En marzo de 1941, el Comité Interamericano de Economía solicita el depósito de una cuota inicial a los países americanos para el fondo de creación del Banco Interamericano. Además, solicita a Ecuador la suscripción mínima de 
cinco acciones de 100.000 dólares $^{2}$ cada una, lo cual correspondía a 7.5 millones de sucres ${ }^{3}$.

Poco después, en diciembre de ese mismo año, Estados Unidos comienza a abordar el tema de seguridad económica para la región. Es así como los americanos exponen algunas medidas, tales como el control de exportaciones para conservar los elementos para fines militares, el incremento de la producción de materiales bélicos, la provisión de materiales necesarios para las economías internas de los países americanos, el mantenimiento de transportes marítimos adecuados, y el control de actividades comerciales y financieras de extranjeros consideradas perjudiciales para las naciones americanas ${ }^{4}$.

\section{¿Neorrealismo en el enfoque de seguridad americano durante la Segunda Guerra Mundial?}

Dentro de las teorías de relaciones internacionales, si se tuviese que elegir una para explicar el enfoque de seguridad americano en el período analizado, la que brinda una explicación más completa es el neorrealismo de Kenneth Waltz, quien analiza las estructuras anárquicas y las balanzas de poder en su libro titulado Teoría de la política internacional (1979). El politólogo sostiene que

(...) las estructuras hacen que las acciones tengan consecuencias que no estaban destinadas a tener. Seguramente la mayoría de los actores lo notarán, y al menos algunos de ellos podrán descubrir por qué. Pueden desarrollar un sentido bastante bueno de cómo funcionan los efectos de las estructuras (Waltz, 1979, p.107)

\footnotetext{
${ }^{2}$ Corresponde al valor de la época, el cual puede ser interpretado, considerando la inflación, a US\$1.724.917,87 de dólares en el 2018, según el Consumer Price Index statistics del Historical Statistics of the United States.

${ }^{3}$ Archivo Histórico del Ministerio de Relaciones Exteriores del Ecuador (en adelante AHMREE), Comunicaciones reservadas dirigidas por la Embajada de Ecuador en los Estados Unidos de América 1937-1940, Washington, 8 de marzo de 1940.

${ }^{4}$ AHMREE, Comunicaciones reservadas dirigidas por la Embajada de Ecuador en los Estados Unidos de América 1937-1940, Washington, 18 de diciembre de 1941.
} 
En vista de la amenaza que supone el Tercer Reich y también el Imperio del Japón para los intereses americanos, desde la década de 1930, los dirigentes estadounidenses tienen bien en claro que debían crear un enfoque de seguridad que les permitiera perfilarse como una potencia. Dicho enfoque combina seguridad y economía, y configura así "estructuras mixtas" dentro de la nación como en el sistema internacional, gracias a un empoderamiento de su economía doméstica que refuerza su influencia económica en el exterior. A diferencia del escenario post-Primera Guerra Mundial, donde los americanos se beneficiaban de un crecimiento económico, en este caso el efecto inesperado de estas estructuras que menciona Waltz, radica en que Estados Unidos se convierte en una potencia global.

Con respecto a la estructura de seguridad y económica que teje los Estados Unidos en América Latina, específicamente en el caso de Ecuador, se puede afirmar que este país ofrece préstamos, consultorías y asistencia económica y el fortalecimiento de las relaciones comerciales interamericanas. La contraparte es que algunas de estas medidas consisten en el control del ingreso de naciones del Eje a Ecuador e intercambio de información de sospechosos, Inteligencia de Señales, Operación Mañana Land ${ }^{5}$ y la presencia del Federal Bureau of Investigation.

\section{Actividades conjuntas entre Ecuador y Estados Unidos en el campo de la seguridad durante la Segunda Guerra Mundial}

Para entender la relación de Estados Unidos con Ecuador, es necesario también conocer cómo los americanos interactúan con los demás países de la región. En el Período de Entreguerras (1919-1939), Estados Unidos maneja una política exterior hacia Latinoamérica denominada la Política del Buen Vecino, la cual consiste en el reforzamiento de los lazos de cooperación intra continental para afianzar el liberalismo, la diplomacia del dólar y evitar la influencia de ideologías consideradas peligrosas desde el Viejo Continente. En 1939, y durante el periodo bélico hasta 1945, la Política del Buen Vecino gira hacia la defensa del hemisferio occidental mediante la cooperación financiera y la asistencia técnica desde Estados Unidos para el

${ }^{5}$ Denominación que recibe la operación de inteligencia de señales llevada a cabo en el Ecuador durante la Segunda Guerra Mundial 
mantenimiento del desarrollo progresivo del continente a pesar de la guerra. Es así que, en 1940, la capacidad de préstamos del Eximbank aumentó de 200 a 700 millones de dólares, ese mismo año inicia el proyecto del Banco Interamericano, y en 1942 se crea el Instituto para Asuntos Interamericanos que brindaba apoyo en programas de desarrollo de agricultura, salud y educación (Alcalde Cardoza, 1997).

En el caso ecuatoriano, las actividades conjuntas realizadas con Estados Unidos se inician con la firma del Acuerdo Marco de Defensa del Hemisferio. Abarca el reforzamiento del ejército y la vigilancia de las comunicaciones; el establecimiento de bases militares en Salinas y en Galápagos; la supervisión del ingreso de ciudadanos de las naciones del Eje y el canje de información de sospechosos; la aplicación de la Inteligencia de Señales (Signal Intelligence) con la Operación Mañana Land y la participación del Federal Bureau of Investigation; y la implementación de la Lista Negra.

La cooperación en seguridad entre ambos países, además, se inscribe en el Acuerdo Marco de Medidas de Cooperación para la Defensa del Hemisferio, firmado en julio de 1940, en la Segunda Reunión de los Ministros de Relaciones Exteriores de las Repúblicas Americanas. Entre las pautas se acuerda que cualquiera de los dos países tiene la facultad de ejecutar operaciones defensivas en las aguas o territorios del otro país, utilizar las instalaciones defensivas, la excepción de impuestos a importaciones del otro país y la vigencia del convenio mientras hubiera alguna amenaza de ataque de un país no americano a uno americano ${ }^{6}$.

De este acuerdo marco se deriva un convenio complementario, firmado el 12 de diciembre de 1941. Este dispone la aceptación del Ecuador para la realización de operaciones de defensa continental estadounidense en la costa ecuatoriana y Galápagos ${ }^{7}$. Asimismo, conlleva una misión naval y una aérea. La Misión Naval duraría cuatro años y estaría a cargo un jefe de misión de rango de teniente comandante y personal naval. El personal ostentaría autoridad sobre los oficiales locales del mismo rango; pero acatarían órdenes del ministro de Defensa ecuatoriano. Entre sus funciones se destacan la

${ }^{6}$ AHMREE, Documentos de la ocupación del Archipiélago de Galápagos 1940-1944, julio, 1940.

${ }^{7}$ AHMREE, Comunicaciones reservadas dirigidas por la Embajada de Ecuador en los Estados Unidos de Norteamérica, Washington, 19 de diciembre de 1941. 
creación y aplicación de programas, estrategias defensivas y el entrenamiento de personal local ${ }^{8}$.

Como parte del acuerdo anterior, Estados Unidos apoya el desarrollo de las fuerzas armadas de los países americanos, a través de la adjudicación de materiales bélicos y municiones. A Ecuador se entregaron US\$17.000.000 en 1941, para el abastecimiento de armamento (balas y fusiles) ${ }^{9}$. Asimismo, el Proyecto de adquisiciones bélicas (Lend Lease Act) de Estados Unidos, aprobado en diciembre de 1941, se orienta al envío de material bélico para medios de transporte, vehículos de combate, infantería, aviación, artillería; además de ingenieros y otros servicios solicitados por el Ministerio de Defensa del Ecuador. Es así que, para febrero de 1945, el material bélico entregado Ecuador fue de US $\$ 5931.935,42$ dólares $^{10}$.

Bajo el argumento de la alianza regional y de contención de cualquier amenaza a la defensa continental, se incluye la censura a las comunicaciones. Tema que fue tratado en la Junta Interamericana de Defensa, originada el 28 de enero de 1942 (Tercera Reunión de Consulta de ministros de Relaciones Exteriores de las 21 Repúblicas Americanas, Río de Janeiro). Dicha Junta conforma una Comisión integrada por técnicos militares, navales y de la Fuerza Aérea, elegidos por las naciones de la Unión Panamericana, con el objetivo de crear estrategias defensivas para el continente americano contra el peligro de las potencias del Eje ${ }^{11}$. Por lo que, en varias resoluciones se dispone la vigilancia de noticias difundidas al interior de los países americanos, para salvaguardar la paz y seguridad continental, y evitar las amenazas a la seguridad y defensa de las democracias americanas (Estrada, 2006, p.30).

Estados Unidos determina en Ecuador las zonas estratégicas de seguridad, basándose en el plan de la zona de seguridad americana. Para ello, establece bases militares en Salinas, como punto fundamental en territorio continental,

\footnotetext{
${ }^{8}$ AHMREE, Comunicaciones dirigidas por la Legación de Estados Unidos de América en el Ecuador , julio-diciembre 1941.

${ }_{9}^{A}$ AHMREE, Comunicaciones dirigidas por la Embajada de Estados Unidos en Ecuador, Quito, 11 de marzo de 1941.

${ }^{10}$ AHMREE, Comunicaciones reservadas dirigidas por la Embajada del Ecuador en los Estados Unidos de Norteamérica, Washington, 24 de diciembre de 1941.

${ }^{11}$ Reseña Histórica, Junta Interamericana de Defensa, https://www.jid.org/?page_id=285
} 
y en Galápagos, como zona táctica en el Océano Pacífico, debido a la preocupación norteamericana sobre una presumible arremetida de fuerzas alemanas por el Canal de Panamá, bajo la sospecha de que parte de dichas fuerzas se encontraban ocultas en la selva amazónica. Es decir que el establecimiento de bases militares en esos espacios permitiría una pronta reacción para proteger el canal, si ocurría un ataque nazi (Lauderbaugh, 2010, pp. 268-270).

La base militar norteamericana en Salinas se vuelve una realidad con el Acuerdo de Salinas, firmado el 24 de enero de 1942. Este autoriza la posesión de la Parroquia de Salinas (provincia de Santa Elena), la edificación de campos de aterrizaje, ranflas de acoderaje, almacenes, entre otros. Se permitía la instauración de cables submarinos, radios y boyas para la ejecución de operaciones aéreas, navales y militares. Asimismo, la inclusión de personal policial estadounidense en funciones internas, además, de la colaboración del cuerpo de Aviación, Marina y Ejército estadounidense. Este acuerdo enfatiza el respeto de la soberanía ecuatoriana, la no concesión de ningún título de propiedad a Estados Unidos, y la jurisdicción ecuatoriana sobre las edificaciones realizadas, después de terminada la amenaza a la seguridad continental y una vez abandonadas las bases ${ }^{12}$.

Por su parte, el establecimiento de la base de Galápagos se concreta con el Acuerdo para el uso y funcionamiento de las áreas defensivas de los Estados Unidos en el Archipiélago de Galápagos, firmado el 2 de febrero de 1942. Se autoriza la instalación de muelles, bodegas, cuarteles y campos de aviación, para acciones áreas, militares y navales. Además, instalaciones submarinas y detectoras en las aguas; cooperación recíproca para acciones terrestres, aéreas (aterrizajes, despegues) y navales (ancladeros, atracaderos) ${ }^{13}$.

Este acuerdo tendría vigencia hasta después de un año de terminado el conflicto bélico. Estados Unidos no solicitaría ningún título de propiedad sobre la construcción, en respeto de la soberanía ecuatoriana; los productos importados por Estados Unidos a la base no serían gravados y el acuerdo

${ }^{12}$ AHMREE, Documentos de la ocupación del Archipiélago de Galápagos 1940-1944, 24 de enero de 1942.

${ }^{13}$ AHMREE, Documentos de la ocupación del Archipiélago de Galápagos 1940-1944, 2 de febrero de 1942. 
debía permanecer en confidencialidad hasta que ambas partes lo denunciaran ${ }^{14}$.

En cuanto a las cuestiones de inteligencia, bajo el marco de la cooperación interamericana, se concretan algunos programas secretos entre Ecuador y Estados Unidos para evitar la injerencia de la Alemania Nazi en territorio americano.

Las medidas de supervisión del ingreso de ciudadanos de las naciones del Eje y el canje de información de sospechosos tienen como objetivo impedir la entrada de ideologías fascistas europeas a América. El 5 de febrero de 1941, la Legación Americana propone el canje de información sobre personal consular o diplomático de Gobiernos no americanos, y de ciudadanos calificados como no gratos por cualquier nación del nuevo continente ${ }^{15}$. Sirva como ejemplo la solicitud de la Legación de los Estados Unidos en agosto de 1942 al Ministerio de Relaciones Exteriores ecuatoriano respecto de tomar las medidas concernientes para la recepción de solicitud de ingreso de Emmy Fenthol, ciudadana alemana, estimada como sospechosa y de su exesposo, Fritz Fenthol, ambos tildados como nazis ${ }^{16}$. Es así que, para marzo de 1943, Ecuador emite las listas del Registro de Extranjeros Sospechosos e Indeseables a la Dirección General de Identificación y Dactiloscopia estadounidense ${ }^{17}$.

A su vez, con relación a la supervisión migratoria de ciudadanos de Alemania, Italia y Japón, Estados Unidos solicita la intensificación de las acciones de las autoridades ecuatorianas para impedir cualquier infiltrado en territorio americano. El 8 de enero de 1943, el Consulado General de los Estados Unidos en Guayaquil juzga la poca severidad en la supervisión de personas que arribaban al aeropuerto de Guayaquil, destaca la envergadura táctica que acarreaba el desembarco de vuelos internacionales, y solicita la aplicación de

${ }^{14}$ AHMREE, Documentos de la ocupación del Archipiélago de Galápagos 1940-1944, 2 de febrero de 1942.

${ }^{15}$ AHMREE, Comunicaciones dirigidas por la Legación de los Estados Unidos de América en el Ecuador enero-junio1941, Quito, 5 de febrero de 1941.

${ }^{16}$ AHMREE, Comunicaciones dirigidas por la Embajada de los Estados Unidos en Ecuador enero- abril 1942, Quito, 4 de agosto de 1942.

${ }^{17}$ AHMREE, Comunicaciones dirigidas por la Embajada de Estados Unidos de América acreditada en el Ecuador Enero- Abril, Quito, 2 de febrero de 1943. 
las restricciones acordadas a los nacionales del Eje que llegasen al terminal aéreo ${ }^{18}$.

La puesta en marcha de programas de inteligencia norteamericanos dentro de Ecuador tiene como objetivo la represión de elementos alemanes infiltrados en América Latina. Acorde con información desclasificada de la Agencia Nacional de Seguridad (National Security Agency), el enfoque de seguridad estadounidense en Latinoamérica se dirige al desenvolvimiento de la inteligencia de señales a manos de la armada, el FBI y el ejército, entre 1939 y 1942, además del avance criptoanalítico e informativo frente a las intercomunicaciones entre agentes del Eje (Mowry, 1989, pp.37-40). Cabe mencionar que dicha acción tiene como antecedente las primeras comunicaciones clandestinas de agentes del Eje en América Latina, que tuvieron lugar en este país, en mayo y junio de 1940, siendo prueba de la existencia de una red radial nazi en Latinoamérica. Es por ello que, bajo el sistema interamericano de cooperación, Ecuador pide a Estados Unidos el envío de equipos técnicos de localización de transmisores de radio ilegales. Además, por solicitud del embajador americano Boaz Long, se solicitan dos receptores de radio y nueve expertos de la Armada (Mowry, 1989, pp. 35-37). Bajo esta línea, en 1941, se peticiona el envío de equipo y personal naval para el entrenamiento de operadores locales en el rastreo de señales. $Y$ el ministro de Guerra del Ecuador autoriza únicamente a los oficiales de la armada norteamericana a la operación de este proyecto, relacionado a la retransmisión de mensajes codificados procedentes de Nauen y Kiel (Alemania) (Mowry, 1989, pp. 37-40).

En 1941 se pone en marcha la Operación Mañana Land, relacionada a la práctica de Inteligencia de Señale ${ }^{19}$, en la que los oficiales estadounidenses Raymond H. Bradford y Harry I. Maltz arriban a Ecuador con la tarea de capacitar a oficiales locales en la búsqueda de estaciones radiales clandestinas pertenecientes al Eje. En 1943, llega a Quito Charles Week, agente de la American Intelligence Service, quien moviliza la estación de inteligencia a Guayaquil, debido a la interferencia en las ondas provocadas por las montañas, y ordena la construcción de antenas de recepción. Este

\footnotetext{
${ }^{18}$ AHMREE, Comunicaciones dirigidas por la Embajada de los Estados Unidos de América acreditada en el Ecuador enero-abril 1943, Quito, 8 de enero de 1943.

${ }^{19}$ Recolección de datos mediante la intercepción de señales entre individuos y señales electrónicas
} 
proyecto fue apoyado por el Ministerio de Defensa ecuatoriano hasta julio de 1943, fecha en la que termina el proyecto (Mowry, 1989, p.53-54).

Para 1941, la labor del FBI se dirige a la capacitación policial, tras la firma de un convenio entre el presidente de Ecuador, Carlos Arroyo del Río, y el Departamento de Estado de Estados Unidos. Ciertos agentes inician actividades encubiertas bajo licencias diplomáticas, y uno es contratado por la Embajada Americana como asistente legal. Este último es un miembro de la cartera de Estado de Arroyo del Río, dándole la facultad al FBI para el exilio de nacionales del Eje domiciliados en Ecuador (Becker, 2016, p.161).

Según datos obtenidos sobre la disposición de los agentes del FBI activos en el Estado ecuatoriano, se conoce que entre marzo y abril de 1943 hay 1 encuestador de plantas industriales en la capital, 16 agentes oficiales, 5 enlaces policiales y 6 agregados legales. Para octubre de ese año, se detectan 21 agentes del FBI en Ecuador, la mayor parte en Guayaquil y Quito, pero también en localidades como Cuenca, y Ambato, debido a la reubicación de algunos ciudadanos del Eje (Becker, 2016, pp.161-162).

El posicionamiento de la inteligencia estadounidense y la apertura del gobierno ecuatoriano a la cooperación con Estados Unidos se traduce en la prohibición de apología nazi, la influencia en los contenidos de la prensa ecuatoriana, y la aprehensión y deportación a campos de trabajo americanos de ciudadanos alemanes y sus aliados. Medidas que afectan a 500 personas (alemanas, ecuatorianas) y firmas instauradas en Ecuador (Estrada, 2006, p.13).

\section{La relación entre Ecuador y Estados Unidos en el campo de la seguridad durante la Segunda Guerra Mundial: ¿juego suma cero o estrategia ganar-ganar?}

La imagen de los Estados Unidos se deteriora por su afán de intervencionismo durante las dos primeras décadas del siglo XX. Es así como los americanos tratan de acercarse nuevamente a los países latinoamericanos con el objetivo de tener aliados en el marco de la Segunda Guerra Mundial; para lo cual convencen a estos países de las ventajas de la integración interamericana con préstamos, asesorías, capacitaciones en diferentes áreas como la seguridad y la defensa entre otras. 
Dentro de este contexto, los americanos fijan su mirada en Ecuador, puesto que ven a este país como un socio estratégico tal vez por su posición geográfica, o bien por sus recursos naturales. Este acercamiento se produce cuando es necesario sumar países como aliados y reforzar la imagen de potencia líder de los Estados Unidos. La pregunta a plantearse es ¿qué tan conveniente fue esta estrategia para ambos actores?

Von Neumann and Morgenstern afirman que el punto central de la teoría económica es el mecanismo complicado de precios y producción, así como de la ganancia y el gasto de resultados los cuales están condicionados por la conducta racional de los individuos (Von Neumann y Morgenstern, 1953, p.8). En contraparte, el campo de las teorías de relaciones internacionales engloba diversos tópicos, desde la cooperación hasta la seguridad cuyo fin primordial es la hegemonía en el sistema internacional. Esto motiva a algunos países a que sumen esfuerzos y trabajen de manera conjunta con algún objetivo en común. El resultado de tal decisión se puede entender desde la teoría del juego. Para Von Neumann y Morgenstern el juego reúne tres condiciones. Primero debe percibirse como la totalidad de las reglas que lo definen. Luego hay que centrarse en las movidas, es decir, los elementos esenciales y que están definidos por la elección del participante. Finalmente, las reglas del juego deben ser claras para evitar alguna confusión con las estrategias de los jugadores (Von Neumann y Morgenstern, 1953, p.49).

Dentro de este contexto Von Neumann y Morgenstern sostienen que un juego de Suma-Cero, o estrategias competitivas, supone que cualquier ganancia para una parte conlleva, en equilibrio, la pérdida correspondiente para la otra (Von Neumann y Morgenstern, 1953, p.84). Según James H. Read, el juego de Suma-Cero puede ser entendido en términos de poder político, considerando que dentro de este juego la cooperación y las asociaciones de poder, y las relaciones conflictivas están entrelazadas (Read, 2012, pp.1618). Para ello recurre a la definición de poder de Robert Dahl (1957), la cual exhibe que "A tiene el poder sobre $B$, en la medida en que puede hacer que B haga algo, que de otra manera no haría" (Dahl, 1957, pp. 203-207).

Es así como el trabajo conjunto entre Ecuador y Estados Unidos en el campo de la seguridad internacional evidencia un juego de Suma-Cero en relación con el poder político. Estados Unidos estrecha las alianzas en el sistema interamericano para agrupar el poder de los pequeños Estados, dentro del conflicto global, y le quita ese poder aliado a los países del Eje. En uso de su poder hegemónico, Estados Unidos influencia al país latinoamericano para la 
firma de tratados y acuerdos que permitieran la presencia de ese poder dentro de territorio ecuatoriano.

En ese marco, Ecuador acata acciones con injerencia política externa y, en cierta medida, cede su soberanía. Esto bajo la aplicación de políticas como la Lista Negra, que era de planificación y organización estadounidense, así como el accionar de agentes del FBI en actividades estatales, y la subsiguiente negación americana al gobierno ecuatoriano sobre los pedidos de compensación a ecuatorianos y alemanes, confinados en campos de trabajo estadounidenses. De esta manera, queda demostrada la ganancia de poder en la política interna de Ecuador a favor de Estados Unidos, y la pérdida de poder (soberano) del país andino.

De la misma manera que las relaciones entre los Estados Unidos y Ecuador durante la Segunda Guerra Mundial se pueden explicar desde una perspectiva de juego Suma-Cero, también se podría afirmar que al finalizar el conflicto bélico, esa relación puede llevar implícita una estrategia GanarGanar. Esta afirmación tiene asidero en el escenario post guerra, donde el statu quo del sistema internacional se modifica, alejándose del eurocentrismo y la multipolaridad, e inaugura una pugna ideológica entre socialismo y liberalismo, encarnada en los Estados Unidos y la Unión Soviética respectivamente. Al ganar los aliados se podría afirmar que la relación entre Estados Unidos y Ecuador durante la Segunda Guerra Mundial fue finalmente una relación Ganar-Ganar. En la teoría del juego, Robert Axelrod (1984) define esta estrategia como la diseñada para que todos los participantes puedan beneficiarse, donde se privilegia el poder de lo colectivo. Con este tipo de estrategia se demuestra que cuando las personas cooperan, se obtienen mejores resultados que cuando no cooperan (Axelrod, 1984, p.170). En el caso de Estados Unidos es la potencia que ejerce un poder directo en Latinoamérica traducido en poder político y económico. Ecuador, por su parte, no solo gana a un aliado, sino que se convierte en socio de la potencia más importante en la Guerra Fría y, con el transcurso de los años, sería su primer socio comercial.

\section{Conclusiones}

La estrategia americana aplicada durante la Segunda Guerra Mundial prioriza las estructuras mixtas, es decir, económicas y de seguridad tanto a nivel interno como externo, a fin de reforzar su hegemonía en el sistema 
internacional. Para lo cual, busca agrupar las capacidades de los otros Estados a su favor, restándoles esa posibilidad a sus enemigos.

Las relaciones entre Ecuador y Estados Unidos en seguridad durante este período incluyen varios factores, desde la alianza económica hasta la cooperación defensiva y de seguridad. Esta última contempla la aplicación de nuevas técnicas como la Inteligencia de Señales y da paso al intervencionismo e influencia política americana dentro del territorio ecuatoriano, dada la presencia de agentes federales en el sistema político del país sudamericano.

Finalmente, de acuerdo con la definición de Juego Suma-Cero enunciada por Von Neumann y Morgenstern (1953) donde sostienen que la ganancia de una parte conlleva a la pérdida correspondiente de la otra y viceversa, se debe mencionar que Estados Unidos se valió del status hegemónico en calidad de potencia emergente durante la Segunda Guerra Mundial, para ganar poder político y militar (bases en Galápagos y Salinas), dentro de territorio latinoamericano a través de Ecuador. Entendiéndose que dicho poder (soberano) es restado a Ecuador y se le atribuye a Estados Unidos, cuya influencia se puede apreciar en la toma de decisiones políticas del país andino. Esto se compensa con los beneficios que alcanza Ecuador en el comercio, la cooperación militar y las capacitaciones entregadas por Estados Unidos, así como la adquisición de financiamiento, estructuras, equipos y formación militar. Al finalizar la Segunda Guerra Mundial y la consecuente eliminación de la Alemania Nazi gracias a la sinergia de esfuerzos de la región, se detecta una estrategia Ganar-Ganar donde Estados Unidos se consolidaría como potencia y Ecuador se beneficiaría con el apoyo americano, siendo el más importante del sistema internacional. Posteriormente el país andino sería considerado como un aliado en la lucha norteamericana contra el socialismo en Latinoamérica, durante la Guerra Fría entre 1946 y 1991.

\section{Bibliografía}

Axelrod, R. (1984). The-Evolution-of-Cooperation. Basic Books.

Alcalde Cardoza, J.(1997). La cooperación interamericana durante la Segunda Guerra Mundial y la integración económica continental. 


Agenda Internacional 4 (8), 63-73.

http://revistas.pucp.edu.pe/index.php/agendainternacional/article/view/ 7180

Becker, M. (2016). La Gloriosa bajo la mirada imperial del gobierno de los Estados Unidos. En S. Cabrera (Ed.) La Gloriosa irevolución que no fue? (pp. 153-169). Universidad Andina Simón Bolívar / Corporación Editora Nacional

Dahl, R (1957). The concept of power. Behavioral Science (BS), 2 (3), 201205. http://dx.doi.org/10.1002/bs.3830020303

Estrada, J. (2006). II Guerra Mundial: Lista Negra en Ecuador. Poligráfica.

Golson, E. (2019). The economics of neutrality in World War II. Vox Eu. Recuperado de https://voxeu.org/article/economics-neutrality-worldwar-ii

Hobsbawm, E. (1999). Historia del Siglo XX. Crítica.

Mowry, D. (1989). German Clandestine Activities in South America in World War II. Office of Archives and History National Security Agency/Central Security Service.

Read, J. (2012). Is Power Zero-Sum or Variable-Sum? Old Arguments and New Beginnings. Journal of Political Power, 5 (1), 5-31. https://doi.org/10.1080/2158379X.2012.659865

Stuart, D. (2008). Creating the National Security State: A history of the law that transformed America. Princeton University Press.

Von Clausewitz, C. (1832). Vom Kriege. Clausewitz-Gesellschaft.

Von Neumann, J. y Morgenstern, O. (1953). Theory of the Game and Economic Behavior. Princeton University Press.

Waltz, K. (1979). Theory of International Politics. McGraw Hill.

Lauderbaugh, G (2010). Estados Unidos y Ecuador durante la Segunda Guerra Mundial: conflicto y convergencia. En B. Zepeda (Comp.). Ecuador: relaciones internacionales a la luz del bicentenario, (pp.265295). FLACSO. 